\title{
A Representation of a Point Symmetric 2-Structure by a Quasi-Domain
}

\author{
Helmut Karzel, Jarosław Kosiorek and Andrzej Matraś \\ Dedicated to Momme Thomsen on the occasion of his 70th birthday
}

\begin{abstract}
In a symmetric 2-structure $\Sigma=\left(P, \mathfrak{G}_{1}, \mathfrak{G}_{2}, \mathfrak{K}\right)$ we fix a chain $E \in \mathfrak{K}$ and define on $E$ two binary operations "+" and ".". Then $(E,+)$ is a K-loop and for $E^{*}:=E \backslash\{o\},\left(E^{*}, \cdot\right)$ is a Bol loop. If $\Sigma$ is even point symmetric then $(E,+, \cdot)$ is a quasidomain and one has the set $\operatorname{Aff}(E,+, \cdot):=\left\{a^{+} \circ b^{\bullet} \mid a \in E, b \in E^{*}\right\}$ of affine permutations. From $\operatorname{Aff}(E,+, \cdot)$ one can reproduce via a "chain derivation" the point symmetric 2-structure $\Sigma$.
\end{abstract}

Mathematics Subject Classification (1991). 51A99.

Keywords. 2-Structure, symmetric chain structure, permutation set, quasi-domain.

\section{Introduction and notations}

There is a one to one correspondence between chain structures $\Sigma=\left(P, \mathfrak{G}_{1}, \mathfrak{G}_{2}\right.$, $\mathfrak{K})$ and permutation sets $(E, \Omega)$ via a germ operation $\Pi_{E}$ and a chain derivation $\kappa$. If a chain $E \in \mathfrak{K}$ is fixed then $\Pi_{E}$ maps each chain $K \in \mathfrak{K}$ onto a permutation of Sym $E$ and then the pair $(E, \Omega)$ with $\Omega:=\Pi_{E}(\mathfrak{K})$ is called the germ of the chain structure $\Sigma$. Then $\Sigma$ is symmetric i.e. for each $K \in \mathfrak{K}$ the reflection $\widetilde{K}$ in $K$ maps any chain $L \in \mathfrak{K}$ onto a chain of $\mathfrak{K}$ - if and only if

$$
\text { (S) } \forall \omega \in \Omega, \omega^{-1} \in \Omega, \omega \circ \Omega \circ \omega \subseteq \Omega \text {. }
$$

And $\Sigma$ is a 2 -structure iff $(E, \Omega)$ is a sharply 2-transitive permutation set. By [1], we know: If the set $E$ is provided with two binary operations "+" and "." such that $(E,+, \cdot)$ is a field or a near field or even a near domain and if for $a \in$ $E, b \in E \backslash\{o\}$ we set " $a^{+}: E \rightarrow E ; x \mapsto a+x$ " and " $b$ ' $: E \rightarrow E ; x \mapsto b \cdot x$ " and 
Aff $(E,+, \cdot):=\left\{a^{+} \circ b^{\bullet} \mid a \in E, b \in E \backslash\{o\}\right\}$ - called the corresponding affine permutation set - then $(E, \Omega)$ with $\Omega:=A f f(E,+, \cdot)$ is a sharply 2-transitive permutation group. Vice versa if $(E, \Omega)$ is a sharply 2-transitive permutation group then $E$ can be provided with two binary operations "+" and "." such that $(E,+, \cdot)$ is a near-domain and $\operatorname{Aff}(E,+, \cdot)$ coincides with $\Omega$. The notion quasi-domain $(Q,+, \cdot)$, introduced by Kist [2], includes both, quasi-fields and near-domains and for a quasi-domain $(Q,+, \cdot)$ the pair $(Q, A f f(Q,+, \cdot))$ is a set of permutations which is sharply 2-transitive under certain conditions (cf.Theorem 1.5). Here we show: If $(E, \Omega)$ with $\Omega:=\Pi_{E}(\mathfrak{K})$ is the germ of a point symmetric 2-structure $\Sigma$ and if $\mathfrak{J}_{E}:=\left\{\omega \in \Omega \mid \omega^{2}=i d \neq \omega\right\}$ then $(E, \Omega)$ satisfies besides $(S)$ the condition

$$
\left(S^{\prime \prime}\right) \quad \Omega \circ \mathfrak{J}_{E} \subseteq \Omega
$$

and $E$ can be provided with binary operations "+" and "." such that $(E,+, \cdot)$ is a quasi domain with $\Omega=\operatorname{Aff}(E,+, \cdot)$. Moreover $(E,+)$ is a K-loop and $(E \backslash\{o\}, \cdot)$ is a Bol loop (see [3]).

\subsection{Chain Structures}

We recall some facts about chain structures $\Sigma=\left(P, \mathfrak{G}_{1}, \mathfrak{G}_{2}, \mathfrak{K}\right)$ (cf. [4,5]). Let $\left(P, \mathfrak{G}_{1}, \mathfrak{G}_{2}, \mathfrak{C}\right)$ be the corresponding maximal chain structure hence $\mathfrak{K} \subseteq \mathfrak{C}$. If $A, B \in \mathfrak{C}, p, q \in P$ let $p q:=[p]_{1} \cap[q]_{2}, p A:=[p]_{1} \cap A, A p:=[p]_{2} \cap A$,

$$
\widetilde{A B}: P \rightarrow P ; p \mapsto(B p)(p A) \text {. }
$$

and $\widetilde{A}:=\widetilde{A A}$. Moreover let $P^{(2)}:=\left\{(a, b) \in P^{2} \mid\{a, b\} \neq\{a b, b a\}\right\}$.

If $E \in \mathfrak{C}$ is fixed then for $A \cdot B:=\widetilde{A B}(E),(\mathfrak{C}, \cdot)=(\mathfrak{C}, \cdot)_{E}$ becomes a group called the maximal chain group.

We set " $A \perp B \Leftrightarrow \widetilde{A}(B)=B \wedge A \neq B$ " (then $A$ and $B$ are called orthogonal), $\left.\mathfrak{C}^{2 \perp}:=\{A, B) \in \mathfrak{C}^{2} \mid A \perp B\right\}, \mathfrak{K}^{2 \perp}:=\mathfrak{C}^{2 \perp} \cap \mathfrak{K}^{2}, A^{\perp}:=\{C \in$ $\mathfrak{C} \mid C \perp A\}$,

$$
(p \perp A):=\{K \in \mathfrak{C} \mid K \perp A \wedge p \in K\}
$$

and if $A \in \mathfrak{K}$,

$$
(p \perp A)_{\mathfrak{K}}:=(p \perp A) \cap \mathfrak{K} .
$$

The chain structures $\left(P, \mathfrak{G}_{1}, \mathfrak{G}_{2}, \mathfrak{K}\right)$ is called

$$
\begin{aligned}
\text { symmetric } & \Leftrightarrow \forall K \in \mathfrak{K}: \widetilde{K}(\mathfrak{K})=\mathfrak{K} \\
2-\text { structure } & \Leftrightarrow \forall a, b \in P,\{a, b\} \neq\{a b, b a\} \exists_{1} K \in \mathfrak{K}: a, b \in K .
\end{aligned}
$$

In [6] we introduced a classification of symmetric 2-structures $\Sigma$ depending whether for $K \in \mathfrak{K}$ and $p \in K$ the cardinality $\left|(p \perp K)_{\mathfrak{K}}\right|$ is $>1$ (class I), is $=0$ (class II) or is $=1$ (class III). In [7] we showed if $\Sigma$ is of class III then any two orthogonal chains intersect (in exactly one point) and called $\Sigma$ point symmetric.

Now we add some formulas concerning maximal chain structures: 
Theorem 1.1. Let $\left(P, \mathfrak{G}_{1}, \mathfrak{G}_{2}, \mathfrak{C}\right)$ be a maximal chain structure, $E \in \mathfrak{C}$ fixed and let $\mathfrak{J}$ denote the set of all involutions of the group $(\mathfrak{C}, \cdot)_{E}$ then:

(1) $\mathfrak{J}=\{C \in \mathfrak{C} \mid C \perp E\}=E^{\perp}$.

(2) $\forall A, B \in \mathfrak{C}: A \perp B \Leftrightarrow A \cdot B^{-1} \in \mathfrak{J}$.

(3) $\forall A, B, C \in \mathfrak{C}: \widetilde{A B}(C)=A \cdot C^{-1} \cdot B$.

\subsection{The Germ of a Chain Structure}

Let $\left(P, \mathfrak{G}_{1}, \mathfrak{G}_{2}, \mathfrak{K}\right)$ be a chain structure and $\left(P, \mathfrak{G}_{1}, \mathfrak{G}_{2}, \mathfrak{C}\right)$ the corresponding maximal chain structure. For $A, B \in \mathfrak{C}$ the map $\widehat{A B}$ is a permutation of $P$ mapping the chain $B$ bijectively onto the chain $A$ and $A$ onto $B$. Therefore the restriction $\widetilde{A B} \circ \widetilde{A B}_{\mid B}$ of the product $\widetilde{A B} \circ \widetilde{A B}$ onto the chain $B$ is a permutation of the set of points of $B$. Fixing a chain $E \in \mathfrak{K}$ we obtain by

$$
\Pi_{E}(\mathfrak{K}):=\left\{\widetilde{K E} \circ \widetilde{K E} \widetilde{\mid E}_{\mid K \in \mathfrak{K}\}}\right.
$$

a set of permutations of the elements of $E$. The permutation set

$$
\Pi_{E}\left(\left(P, \mathfrak{G}_{1}, \mathfrak{G}_{2}, \mathfrak{K}\right)\right):=\left(E, \Pi_{E}(\mathfrak{K})\right)
$$

is called the germ of the chain structure $\left(P, \mathfrak{G}_{1}, \mathfrak{G}_{2}, \mathfrak{K}\right)$ in $E$.

We remark $\Pi_{E}(\mathfrak{C})=S y m E$.

Conversely let $(E, \Omega), \Omega \subseteq$ SymE be a permutation set. We set:

$$
\begin{aligned}
& P:=E \times E, \mathfrak{G}_{1}:=\{\{x\} \times E \mid x \in E\}, \mathfrak{G}_{2}:=\{E \times\{x\} \mid x \in E\}, \\
& \forall \omega \in \Omega, \kappa(\omega):=\{(x, \omega(x)) \mid x \in E\}, \kappa(\Omega):=\{\kappa(\omega) \mid \omega \in \Omega\} .
\end{aligned}
$$

Then $\kappa((E, \Omega)):=\left(P, \mathfrak{G}_{1}, \mathfrak{G}_{2}, \kappa(\Omega)\right)$ is a chain structure called chain derivation of the permutation set $(E, \Omega)$. We have:

Theorem 1.2. (1) If $\left(P, \mathfrak{G}_{1}, \mathfrak{G}_{2}, \mathfrak{K}\right)$ is a chain structure, $E \in \mathfrak{K}$ then $\kappa\left(\Pi_{E}\left(P, \mathfrak{G}_{1}, \mathfrak{G}_{2}, \mathfrak{K}\right)\right)=\left(P, \mathfrak{G}_{1}, \mathfrak{G}_{2}, \mathfrak{K}\right)$

(2) If $(E, \Omega)$ is a permutation set then $\Pi_{E}(\kappa(E, \Omega))=(E, \Omega)$.

(3) The map $\Pi_{E}$ is an isomorphism from the group $(\mathfrak{C}, \cdot)_{E}$ onto the symmetric group (Sym E, ०).

Now we apply the germ operation $\Pi_{E}$ on certain sets of chains contained in $\mathfrak{K}$ or in $\mathfrak{C}$.

Theorem 1.3. Let $E \in \mathfrak{K}$ and $o \in E$ be fixed, let $\mathfrak{J}_{E}$ denote the set of all involutions of $($ Sym $E, \circ)$ and let $\Omega:=\Pi_{E}(\mathfrak{K})$. Then:

(1) $\Pi_{E}(\mathfrak{J})=\mathfrak{J}_{E}$ and $\Pi_{E}(E)=i d \in \Omega$.

(2) If $\mathfrak{K}_{o}:=\{K \in \mathfrak{K} \mid o \in K\}$ and $\Omega_{o}:=\{\omega \in \Omega \mid \omega(o)=o\}$ then $\Pi_{E}\left(\mathfrak{K}_{o}\right)=$ $\Omega_{O}$.

(3) Let $\mathfrak{N}_{\mathfrak{C}}\left(E^{\perp}\right):=\left\{C \in \mathfrak{C} \mid C \cdot E^{\perp} \cdot C^{-1}=E^{\perp}\right\}$ be the normalizer of $E^{\perp}$ in the group $(\mathfrak{C}, \cdot)$ and $\mathfrak{N}_{S y m}\left(\mathfrak{J}_{E}\right):=\left\{\sigma \in\right.$ Sym $\left.E \mid \sigma \circ \mathfrak{J}_{E} \circ \sigma^{-1}=\mathfrak{J}_{E}\right\}$ then $\Pi_{E}\left(\mathfrak{N}_{\mathfrak{C}}\left(E^{\perp}\right)\right)=\mathfrak{N}_{\text {Sym }}\left(\mathfrak{J}_{E}\right)$.

(4) If $A, B \in \mathfrak{C}$ and $\alpha:=\Pi_{E}(A), \beta:=\Pi_{E}(B)$ then " $A \perp B \Leftrightarrow \alpha^{-1} \circ \beta \in \mathfrak{J}_{E}$ "

(5) Let $p \in P, A \in \mathfrak{C}$ and $\alpha:=\Pi_{E}(A)$ then: " $p \in A \Leftrightarrow \alpha(p E)=E p$ " and "Fix $\alpha=A \cap E$ ". 
(6) Let $p \in P, A \in \mathfrak{K}$ and $\alpha:=\Pi_{E}(A)$ then

$$
\begin{aligned}
& \Pi_{E}\left((p \perp A)_{\mathfrak{K}}\right)=\left\{\beta \in \Omega \mid \beta(p E)=E p \wedge \alpha^{-1} \circ \beta \in \mathfrak{J}_{E}\right\} \\
& \Pi_{E}\left((p \perp E)_{\mathfrak{K}}\right)=\left\{\beta \in \Omega \cap \mathfrak{J}_{E} \mid \beta(p E)=E p\right\} \\
& \Pi_{E}\left((o \perp E)_{\mathfrak{K}}\right)=\left\{\beta \in \Omega \cap \mathfrak{J}_{E} \mid o \in \text { Fix } \beta\right\}
\end{aligned}
$$

(7) In the case of a symmetric 2-structure we have the following translations: Let $A, B \in \mathfrak{K}$ and $\alpha:=\Pi_{E}(A), \beta:=\Pi_{E}(B)$, then:

$A \perp B \Leftrightarrow A \cdot B^{-1} \in \mathfrak{J} \Leftrightarrow \alpha \circ \beta^{-1} \in \mathfrak{J}_{E}$ $A \cap B \neq \emptyset \Leftrightarrow$ Fix $\alpha \circ \beta^{-1} \neq \emptyset$

(Droping and erecting the perpendicular) $\forall \omega \in \Omega \forall a, b \in E \exists \lambda \in \mathfrak{J}_{E} \circ \omega$ with $\lambda(a)=b$.

\subsection{Translation of Properties}

By Theorem 1.2. each property of a chain structure is rendered to a property of the germ and vice versa. So we have:

Theorem 1.4. Let $\left(P, \mathfrak{G}_{1}, \mathfrak{G}_{2}, \mathfrak{K}\right)$ be a chain structure, $E \in \mathfrak{K}$ and $(E, \Omega):=$ $\Pi_{E}\left(P, \mathfrak{G}_{1}, \mathfrak{G}_{2}, \mathfrak{K}\right)$ the germ. Then:

(1) $\left(P, \mathfrak{G}_{1}, \mathfrak{G}_{2}, \mathfrak{K}\right)$ is a 2-structure $\Leftrightarrow(E, \Omega)$ is a sharply 2-transitive permutation set.

(2) $\left(P, \mathfrak{G}_{1} \cup \mathfrak{G}_{2} \cup \mathfrak{K}\right)$ is an affine plane $\Leftrightarrow(E, \Omega)$ is a sharply 2-transitive permutation set and $\forall \alpha \in \Omega, \forall a, b \in E$ with $\alpha(a) \neq b, \exists_{1} \beta \in \Omega$ such that $\beta(a)=b$ and Fix $\left(\beta \circ \alpha^{-1}\right)=\emptyset$.

(3) $\left(P, \mathfrak{G}_{1}, \mathfrak{G}_{2}, \mathfrak{K}\right)$ is symmetric (i.e. $\left.\forall K \in \mathfrak{K}: \widetilde{K}(\mathfrak{K})=\mathfrak{K}\right) \Leftrightarrow$ (cf. [4])

$$
\begin{aligned}
& \forall K \in \mathfrak{K}: K \cdot \mathfrak{K} \cdot K=\mathfrak{K} \wedge \mathfrak{K}^{-1}=\mathfrak{K} \Leftrightarrow \\
& (S) \Omega=\Omega^{-1} \wedge \forall \omega \in \Omega: \omega \circ \Omega \circ \omega=\Omega .
\end{aligned}
$$

(4) $\left(P, \mathfrak{G}_{1}, \mathfrak{G}_{2}, \mathfrak{K}\right)$ is a symmetric 2-structure $\Leftrightarrow(E, \Omega)$ is a sharply 2-transitive permutation set satisfying $(S)$ and $i d \in \Omega$.

A permutation set $(E, \Omega)$ satisfying the condition $(\mathrm{S})$ shall be also called symmetric.

\subsection{Quasi-Domains and Their Affine Permutations}

In order to have a common concept which includes both, near-domains and quasi-fields, Kist (cf. [2]) introduced the notion quasi-domain. That is a set $(Q,+, \cdot)$ provided with two binary operations such that the following conditions are satisfied:

(1) $(Q,+)$ is a loop (o denotes the neutral element; let $Q^{*}=Q \backslash\{o\}$ ).

(2) $\forall a, b \in Q: a+b=o \Rightarrow b+a=o$.

(3) $\left(Q^{*}, \cdot\right)$ is a loop (let $e$ denote the neutral element of $\left(Q^{*}, \cdot\right)$ ).

(4) $\forall a, b, c \in Q: a(b+c)=a b+a c$. 
(5) $\forall a \in Q: o \cdot a=o$.

(6) $\forall a, b \in Q \exists d_{a, b} \in Q$ such that $\forall x \in Q: a+(b+x)=(a+b)+d_{a, b} x$.

By (Q.4), " $\forall x \in Q: x+x=o \Leftrightarrow e+e=o "$.

Therefore we say: " $(Q,+, \cdot)$ has characteristic 2 if $e+e=o$ " (expressed by $\operatorname{char}(Q)=2)$ and " $(Q,+, \cdot)$ has characteristic $\neq 2$ if $e+e \neq o$ " (expressed by $\operatorname{char}(Q) \neq 2)$.

Remark 1 . If $(Q,+, \cdot)$ is a quasi-domain then by $(6),(Q,+)$ is an $A_{l}$-loop (cf. [3] p.35). $(Q,+, \cdot)$ is a quasi-field if $(Q,+)$ is a group, a near-domain if $\left(Q^{*}, \cdot\right)$ is a group and a near-field if $(Q,+)$ and $\left(Q^{*}, \cdot\right)$ are groups.

If $(Q,+, \cdot)$ is a quasi-domain then for each $a \in Q$ and for each $b \in Q^{*}$ the maps

$$
\text { " } a^{+}: Q \rightarrow Q ; x \mapsto a+x " \text { and " } b \bullet: Q \rightarrow Q ; x \mapsto b \cdot x "
$$

are permutations of Sym $Q$ - we set $Q^{+}:=\left\{a^{+} \mid a \in Q\right\}$ and $\left(Q^{*}\right)^{\bullet}:=\left\{b^{\bullet} \mid b \in\right.$ $\left.Q^{*}\right\}$ - and the elements of the set

$$
\Gamma:=\operatorname{Aff}(Q,+, \cdot):=\left\{a^{+} \circ b^{\bullet} \mid a \in Q, b \in Q^{*}\right\}=Q^{+} \circ\left(Q^{*}\right)^{\bullet}
$$

are called affine permutations of the quasi-domain $(Q,+, \cdot)$.

By [2] Theorems (1.18), (1.20) and (1.21) we have:

Theorem 1.5 [Kist [2]]. Let $(Q,+, \cdot)$ be a quasi-domain, $\Gamma:=\operatorname{Aff}(Q,+, \cdot)$ the corresponding affine permutation set, $\Gamma_{o}:=\{\gamma \in \Gamma \mid \gamma(o)=o\}$ and

$\Lambda:=Q^{+} \circ \Gamma_{o} \circ Q^{+}$. Then:

(1) $Q^{+} \circ \operatorname{Aut}(Q,+)$ is a group.

(2) $\Gamma=Q^{+} \circ \Gamma_{o}=\Gamma_{o} \circ Q^{+}$and $\left(Q^{+}\right)^{-1}=Q^{+}$.

(3) $\{(\gamma(o), \gamma(e)) \mid \gamma \in \Gamma\}=\left(\begin{array}{l}Q \\ 2\end{array}\right)$.

(4) $\forall p \in Q^{*}, \forall(a, b) \in\left(\begin{array}{c}Q \\ 2\end{array}\right) \exists_{1} \gamma \in \Gamma$ with $\gamma(o)=a$ and $\gamma(p)=b$.

(5) $\Lambda$ acts 2-transitive on $Q$.

(6) The following three statements are equivalent:

"(a) $\forall a . b, c, x \in Q: c\left(d_{a, b} x\right)=\left(c d_{a, b}\right) x ", "$ (b) $\Lambda=\Gamma$ " and

"(c) $(Q, \Lambda)$ is a sharply 2-transitive permutation set".

(7) The following statements are equivalent:

"(a) $a b=e \Rightarrow \forall x \in Q: a(b x)=x ", ~ "(b) \Gamma=\Gamma^{-1}$ ".

(8) The following statements are equivalent $([2$, page 35$])$ :

"( $a) \forall x \in Q:(-e) x=x(-e)$ ", "(b) $\forall a, b \in Q:(-a) b=-a b "$.

(9) (7) implies (8).

Proof. (1) Let $a, b \in Q, \alpha, \beta \in \operatorname{Aut}(Q,+)$. Then $\left(a^{+} \circ \alpha\right) \circ\left(b^{+} \circ \beta\right)=(a+$ $\alpha(b))^{+} \circ d_{a, \alpha(b)}^{\bullet} \circ \alpha \circ \beta$. By (Q.4), $d_{a, \alpha(b)}^{\bullet} \in \operatorname{Aut}(Q,+)$ hence $\left(a^{+} \circ \alpha\right) \circ\left(b^{+} \circ\right.$ $\beta) \in Q^{+} \circ \operatorname{Aut}(Q,+)$ and $\left(a^{+} \circ \alpha\right)^{-1}=\left(\alpha^{-1}(-a)\right)^{+} \circ\left(\alpha^{-1} \circ\left(\left(d_{a,-a}\right)^{\bullet}\right)^{-1}\right) \in$ $Q^{+} \circ \operatorname{Aut}(Q,+)$.

Remark 2. Kühlbrandt used for his investigations [8] a more restricted concept of quasi-domain by claiming also the property $(6$ (a)) of Theorem 1.5. as an axiom. 
He considered the following condition for sharply 2-transitive permutation set $(E, \Omega)$ :

(*) $\mathfrak{J}_{E} \cdot \Omega=\Omega \cdot \mathfrak{J}_{E}=\Omega$ and every $\omega \in \Omega$ which interchanges two distinct elements of $E$ is an involution.

We recall the following result of Kühlbrandt:

Theorem 1.6 (Kühlbrandt [8]).

(1) Let $(\Omega, E)$ be a sharply 2-transitive permutation set satisfying condition (*). Then an addition and multiplication can be defined in $E$ such that $(E,+, \cdot)$ is a quasi-domain satisfying the conditions (6)(a) and (8)(b) of Theorem 1.5. Furthermore $\Omega$ is the set of affine mappings of $(E,+, \cdot)$.

(2) Let $(E,+, \cdot)$ be a quasi-domain satisfying the condition (6)(a) of Theorem 1.5 and let $\Omega$ be the set of affine mappings of $(E,+, \cdot)$. Then:

(a) $(*)$ is equivalent to the condition (8)(b) of Theorem 1.5.

(b) $(S)$ is equivalent to the condition $\forall a, b, x \in E: a(b(a x))=(a(b a)) x$ (Bol-identity).

Comparing the results of Kist and Kühlbrandt we get:

Corollary 1.7. If a quasi-domain $(E,+, \cdot)$ satisfies the property $(6(a))$ of Theorem 1.5 and the Bol-identity then $(E,+, \cdot)$ satisfies ( $7(a))$ of Theorem 1.5.

Proof. By Theorem 1.6(2)(b) the set of affine mappings of the quasi-domain satisfies $(S)$ hence in particular it satisfies condition $(7)(\mathrm{b})$ of Theorem 1.5.

\section{Sharply 2-Transitive Permutation Sets}

In this part let $(E, \Omega)$ be a sharply 2-transitive permutation set. Then for $\omega \in \Omega$ also $\left(E, \omega^{-1} \circ \Omega\right)$ is a sharply 2 -transitive permutation set. Therefore we assume for $(E, \Omega)$ without loss of generality, $i d \in \Omega$. For $(a, b) \in\left(\begin{array}{c}E \\ 2\end{array}\right)$ let $(a \leftrightarrow b)$ denote the uniquely determined permutation of $\Omega$ interchanging $a$ and $b$ and let $\mathfrak{J}_{E}^{\prime}:=\left\{(a \leftrightarrow b) \mid(a, b) \in\left(\begin{array}{c}E \\ 2\end{array}\right)\right\}$. If $\mathfrak{J}_{E}$ denotes the set of all involutions in $\Omega$ then $\mathfrak{J}_{E} \subseteq \mathfrak{J}_{E}^{\prime}$. For $a \in E$ let $E^{a}:=E \backslash\{a\},\left(a \leftrightarrow E^{a}\right):=\left\{(a \leftrightarrow x) \mid x \in E^{a}\right\}$, $\tilde{a}^{\prime}:=\left\{\alpha \in \mathfrak{J}_{E}^{\prime} \mid \alpha(a)=a\right\}$ and $\tilde{a}:=\left\{\alpha \in \mathfrak{J}_{E} \mid \alpha(a)=a\right\}$.

\subsection{Two Binary Operations}

We set $E_{f}:=\{p \in E \mid \tilde{p} \neq \emptyset\}$. If $E_{f} \neq \emptyset$ let $o \in E_{f}$ be fixed and let $o^{\circ} \in \tilde{o}$, if $E_{f}=\emptyset$ let any $o \in E$ be fixed and let $o^{\circ}:=i d$. For each $p \in E \backslash\{o\}$ let $p^{\circ}:=(o \leftrightarrow p)$ and $p^{+}:=p^{\circ} \circ o^{\circ}$. Then $\left(E, E^{\circ}\right)$ with $E^{\circ}:=\left\{p^{\circ} \mid p \in E\right\}$ is a reflection structure (cf. e.g. [9] Sect. 6.) and in $E$ we define an addition "+" by:

$$
+: E \times E \rightarrow E ;(a, b) \mapsto a+b:=a^{+}(b) .
$$

Then by $[9] 2.3 .,(E,+)$ is a left loop - called the loop-derivation of $\left(E, \mathfrak{J}_{E}^{\prime}\right)$ in $o-$. 
If $\mathfrak{J}_{E}^{\prime}=\mathfrak{J}_{E}=E^{\circ}$ and if $\mathfrak{J}_{E}$ is invariant, i.e. $\forall \alpha \in \mathfrak{J}_{E}: \alpha \circ \mathfrak{J}_{E} \circ \alpha=\mathfrak{J}_{E}$, then $(E,+)$ is a $\mathrm{K}$ - loop.

Now we fix a further element $e \in E^{*}:=E \backslash\{o\}$ and for each $a \in E^{*}$ let $a^{\bullet} \in \Omega$ be the element uniquely determined by $a^{\bullet}(o)=o$ and $a^{\bullet}(e)=a$. In $E$ we define a multiplication "." by:

$$
\because E \times E \rightarrow E ;(a, b) \mapsto a \cdot b:=\left\{\begin{array}{c}
a^{\bullet}(b), a \neq o \\
o, a=o
\end{array} .\right.
$$

Then $e$ is the neutral element of $(E, \cdot)$ and if $a, b \in E^{*}$ then the equation $a \cdot x=b$ has the unique solution $x=\left(a^{\bullet}\right)^{-1}(b)$ and for $x \cdot a=b$ let $\xi \in \Omega$ uniquely determined by $\xi(o)=o$ and $\xi(a)=b$. Then $x:=\xi(e)$ is the unique solution of $x \cdot a=b$. Therefore $\left(E^{*}, \cdot\right)$ is a loop.

The triple $(E,+, \cdot)$ we call the double-loop-derivation of the sharply 2transitive permutation set $(E, \Omega)$ in the points $(o, e) \in\left(\begin{array}{c}E \\ 2\end{array}\right)$.

\subsection{Symmetric Permutation Sets}

From now on let $(E, \Omega)$ be a sharply 2-transitive symmetric permutation set and let $(E,+, \cdot)$ be the corresponding double-loop-derivation in two points $(o, e) \in\left(\begin{array}{c}E \\ 2\end{array}\right)$.

Theorem 2.1. If $(E, \Omega)$ is symmetric then for each $\omega \in \Omega$ the group $\langle\omega>$ generated by the element $\omega$ is a subset of $\Omega$ and we have:

(1) $\mathfrak{J}_{E}=\mathfrak{J}_{E}^{\prime}$.

(2) $\mathfrak{J}_{E}$ is invariant, i.e. $\forall \alpha \in \mathfrak{J}_{E}: \alpha \circ \mathfrak{J}_{E} \circ \alpha=\mathfrak{J}_{E}$, i.e. $\mathfrak{J}_{E} \subseteq \mathfrak{N}_{\text {Sym }}\left(\mathfrak{J}_{E}\right)$.

(3) $\forall \alpha \in \mathfrak{J}_{E}: \mid$ Fix $\alpha \mid \leq 1$.

(4) $\forall(a, b) \in\left(\begin{array}{c}E \\ 2\end{array}\right)$ the map

$$
(a \leftrightarrow b)^{i}: \tilde{a} \rightarrow \tilde{b} ; \xi \mapsto(a \leftrightarrow b) \circ \xi \circ(a \leftrightarrow b)
$$

is a bijection hence $|\tilde{a}|=|\tilde{b}|$.

(5) If $\exists \alpha \in \mathfrak{J}_{E}$ with Fix $\alpha \neq \emptyset$ then: $\forall b \in E: \tilde{b} \neq \emptyset$.

(6) If $(E, \Omega)$ satisfies the condition

$\left(S^{\prime}\right) \forall \omega \in \Omega: \omega \circ \mathfrak{J}_{E} \circ \omega^{-1} \subseteq \Omega$ then:

(a) $\forall a \in E:|\tilde{a}| \leq 1$.

(b) All elements of $\mathfrak{J}_{E}$ are conjugated in $\Omega$, i.e. $\forall \alpha, \beta \in \mathfrak{J}_{E} \exists \omega \in \Omega$ : $\beta=\omega \circ \alpha \circ \omega^{-1}$.

(c) $\forall(a, b) \in\left(\begin{array}{c}E \\ 2\end{array}\right), \forall \omega \in \Omega:(\omega(a) \leftrightarrow \omega(b))=\omega \circ(a \leftrightarrow b) \circ \omega^{-1}$ hence $\forall \alpha, \beta \in \mathfrak{J}_{E}:|F i x \alpha|=|F i x \beta|$ and $" \forall \omega \in \Omega: \omega \circ \mathfrak{J}_{E} \circ \omega^{-1}=\mathfrak{J}_{E}$ ".

Proof. (1) Let $(a, b) \in\left(\begin{array}{c}E \\ 2\end{array}\right)$ and $\omega:=(a \leftrightarrow b)$ then $\omega^{3} \in \Omega$ and $\omega^{3}(a)=$ $b, \omega^{3}(b)=a$ hence $\omega^{3}=(a \leftrightarrow b)=\omega$ and so $\omega^{2}=i d$. Therefore $\mathfrak{J}_{E}=\mathfrak{J}_{E}^{\prime}$.

(2) Let $\alpha, \beta \in \mathfrak{J}_{E}$ then $\alpha \circ \beta \circ \alpha$ is an involution and by $\omega \circ \Omega \circ \omega=\Omega$ we have $\alpha \circ \beta \circ \alpha \in \mathfrak{J}_{E}$.

(3) Since $(E, \Omega)$ is sharply 2 -transitive and since $i d \in \Omega, \mid$ Fix $\alpha \mid \geq 2$ would imply $\alpha=i d$. 
(4) Let $\alpha \in \tilde{a}$. By (1), $(a \leftrightarrow b)$ and $\beta:=(a \leftrightarrow b) \circ \alpha \circ(a \leftrightarrow b)$ are involutions contained in $\mathfrak{J}_{E}$ and Fix $\beta=b$ hence $\beta \in \tilde{b}$.

(5) By (3), Fix $\alpha$ consists of a single point $a$ hence $\alpha \in \tilde{a}$. By (4), $|\tilde{b}|=|\tilde{a}| \geq 1$.

(6) A proof of (a) and (b) is given in [8] $§ 3(3.1),(3.2),(3.4)$.

According to Theorem 2.1.(4), there are three types of sharply 2-transitive symmetric permutation sets $(E, \Omega)$ possible, depending whether for $o \in E$ we have: $|\tilde{o}|>1$ (Class I), $|\tilde{o}|=0$ (Class II) or $|\tilde{o}|=1$ (Class III) and from Theorem 1.3. (4) we obtain: If $(E, \Omega):=\Pi_{E}(\Sigma)$ is the germ of a symmetric 2-structure $\Sigma$ then $\Sigma$ and $(E, \Omega)$ are in the same class. If $(E, \Omega)$ belongs to class III then $\forall \alpha \in \Omega \cap \mathfrak{J}_{E},|F i x \alpha|=1$, so symmetric 2 -structures of class III we also called point symmetric.

According to Theorem 2.1. (1) and (2), we have:

Theorem 2.2. If $(E, \Omega)$ is of class II or III then $(E,+)$ is a K-loop and:

(1) If $(E, \Omega)$ is of class III then:

(a) $\forall a \in E,|\tilde{a}|=1$ and the map $\sim: E \rightarrow \tilde{E}:=\{\tilde{x} \mid x \in E\} ; x \mapsto \tilde{x}$ is a bijection.

(b) $\tilde{E}=\mathfrak{J}_{E}$.

(c) Any two points $a, b \in E$ have exactly one midpoint $m \in E$, i.e. there is exactly one involution $\mu \in \mathfrak{J}_{E}$ with Fix $\mu=m$ and $\mu(a)=b$.

(d) The K-loop $(E,+)$ is uniquely 2-divisible.

(2) If $(E, \Omega)$ satisfies $\left(S^{\prime}\right)$ then

$\Omega_{o}:=\{\omega \in \Omega \mid \omega(o)=o\} \subseteq \operatorname{Aut}(E,+)$.

Proof. (1) Follows from [7, Theorem 2.4] and $[9, \mathbf{6 . 1}]$.

(2) Let $\gamma \in \Omega_{o}$ and $a, b \in E$. Then by $\gamma(o)=o$ and Theorem 2.1.(6)(c), $\gamma \circ a^{+}=\gamma \circ(o \leftrightarrow a) \circ o^{o}=\gamma \circ(o \leftrightarrow a) \circ \gamma^{-1} \circ \gamma \circ o^{o} \circ \gamma^{-1} \circ \gamma=$ $(\gamma(o) \leftrightarrow \gamma(a)) \circ \gamma(o)^{o} \circ \gamma=(o \leftrightarrow \gamma(a)) \circ o^{o} \circ \gamma=(\gamma(a))^{+} \circ \gamma$. Therefore $\gamma(a+b)=\gamma \circ a^{+}(b)=(\gamma(a))^{+} \circ \gamma(b)=\gamma(a)+\gamma(b)$, i.e. $\gamma$ is an automorphism of $(E,+)$.

Remark 3. If $(E, \Omega)$ is of class I then $E^{\circ} \neq \mathfrak{J}_{E}$ and $E^{\circ}$ is not invariant. Thus $(E,+)$ is only a left loop.

Together with Theorem 2.2. we have the result:

Theorem 2.3. Let $(E, \Omega)$ be of class II or III, then $(E,+, \cdot)$ has the properties:

(1) $(E,+)$ is a K-loop and $\left(E^{*}, \cdot\right)$ is a Bol loop, i.e. $\forall a, b \in E^{*}, a^{\bullet} \circ b^{\bullet} \circ a^{\bullet}=$ $(a \cdot(b \cdot a))^{\bullet}$.

(2) If $(E, \Omega)$ satisfies the condition

$\left(S^{\circ}\right)$ For $\alpha, \beta, \gamma, \delta \in \mathfrak{J}_{E} \cup\{i d\}$ with Fix $(\alpha \circ \beta \circ \gamma \circ \delta) \neq \emptyset: \alpha \circ \beta \circ \gamma \circ \delta \in \Omega$ then

$\forall a, b \in E \exists d_{a, b} \in E$ such that $\forall x \in E: a+(b+x)=(a+b)+d_{a, b} \cdot x$. 
(3) If $(E, \Omega)$ satisfies $\left(S^{\prime}\right)$ then $(E,+, \cdot)$ is left-distributive, i.e. $\forall a, b, c \in E$ : $a \cdot(b+c)=a \cdot b+a \cdot c$.

(4) If $(E, \Omega)$ satisfies the condition

$\left(S^{\prime \prime}\right) \Omega \circ \mathfrak{J}_{E} \subseteq \Omega$

then:

(a) $(E, \Omega)$ satisfies also $\left(S^{\prime}\right),\left(S^{o}\right)$ and $\mathfrak{J}_{E} \circ \Omega \subseteq \Omega$.

(b) The group $\left\langle E^{+}>\right.$generated by $E^{+}:=\left\{p^{+} \mid p \in E\right\}$ is a subset of $\Omega$.

(c) $\forall a, b \in E \exists d_{a, b} \in E$ such that $\forall x \in E: a+(b+x)=(a+b)+d_{a, b} \cdot x$ and moreover $c^{\bullet} \circ d_{a, b}^{\bullet}=d_{c a, c b}^{\bullet} \circ c^{\bullet}$ for $c \in E^{*}$.

(d) $\forall a \in E, \forall b \in E^{*}: a^{+} \circ b^{\bullet} \in \Omega$.

(e) $\forall \omega \in \Omega \exists a \in E, b \in E^{*}$ with $\omega=a^{+} \circ b^{\bullet}$.

(f) $\forall a . b, c, x \in E: c\left(d_{a, b} x\right)=\left(c d_{a, b}\right) x$.

(g) $a b=e \Rightarrow \forall x \in E: a(b x)=x$.

Proof. (1) $a^{\bullet} \circ b^{\bullet} \circ a^{\bullet} \in a^{\bullet} \circ \Omega \circ a^{\bullet} \subseteq \Omega$, by (S), $a^{\bullet} \circ b^{\bullet} \circ a^{\bullet}(o)=o$ and $a^{\bullet} \circ b^{\bullet} \circ a^{\bullet}(e)=a \cdot(b \cdot a)$. Hence $a^{\bullet} \circ b^{\bullet} \circ a^{\bullet}=(a \cdot(b \cdot a))^{\bullet}$.

(2) Since $(E,+)$ is a K-loop we have $a+(b+x)=a^{+} \circ b^{+}(x)=(a+b)^{+} \circ \delta_{a, b}(x)$ where $\delta_{a, b}=\left((a+b)^{+}\right)^{-1} \circ a^{+} \circ b^{+} \in \operatorname{Aut}(E,+)$ hence $\delta_{a, b}(o)=o$ and $\delta_{a, b}=o^{\circ} \circ(a+b)^{\circ} \circ a^{\circ} \circ o^{\circ} \circ b^{\circ} \circ o^{\circ}$ with $o^{\circ},(a+b)^{\circ}, a^{\circ},\left(o^{\circ} \circ b^{\circ} \circ o^{\circ}\right) \in$ $\left(\mathfrak{J}_{E} \cup\{i d\}\right)$. Therefore by $\left(S^{o}\right), \delta_{a, b} \in \Omega_{o}$. If $d_{a b}:=\delta_{a, b}(e)$ then $d_{a b}^{\bullet}=\delta_{a, b}$ and $a+(b+x)=(a+b)+d_{a b} \cdot x$.

(3) We may assume, $a \neq o$ then $a^{\bullet} \in \Omega_{o}$ and by Theorem 2.2. (3), $a^{\bullet} \in$ $\operatorname{Aut}(E,+)$ hence $a \cdot(b+c)=a^{\bullet}(b+c)=a^{\bullet}(b)+a^{\bullet}(c)=a \cdot b+a \cdot c$.

(4) a) Let $\omega \in \Omega$ hence by Theorem 2.1., $\omega^{2} \in \Omega$ and so $\omega^{2} \circ \mathfrak{J}_{E} \subseteq \Omega$. This is equivalent to $\omega \circ \mathfrak{J}_{E} \circ \omega^{-1} \subseteq \omega^{-1} \circ \Omega \circ \omega^{-1} \subseteq \Omega$.

(4) b) Since $p^{+}=p^{\circ} \circ o^{\circ} \in \mathfrak{J}_{E} \circ \mathfrak{J}_{E} \subseteq \Omega \circ \mathfrak{J}_{E} \subseteq \Omega$ and $a^{+} \circ b^{+} \in \Omega \circ b^{\circ} \circ o^{\circ} \subseteq$ $\left(\Omega \circ \mathfrak{J}_{E}\right) \circ \mathfrak{J}_{E} \subseteq \Omega \circ \mathfrak{J}_{E} \subseteq \Omega$ we obtain by induction, $\left\langle E^{+}\right\rangle \subseteq \Omega$.

(4) c) Since $(E,+)$ is a K-loop we have $a+(b+x)=a^{+} \circ b^{+}(x)=(a+$ $b)^{+} \circ \delta_{a, b}(x)$ where by b) $\delta_{a, b}=\left((a+b)^{+}\right)^{-1} \circ a^{+} \circ b^{+} \in \Omega$. Since $\delta_{a, b}(o)=o, \delta_{a, b} \in \Omega_{o} \subseteq \operatorname{Aut}(E,+)$ (cf. Theorem 2.2. (3)) and therefore if $d_{a b}:=\delta_{a, b}(e)$ then $d_{a b}^{\bullet}=\delta_{a, b}$ and $a+(b+x)=(a+b)+d_{a b} \cdot x$. Moreover by (3) and (4) a), $c^{\bullet} \circ a^{+} \circ b^{+}(x)=c \cdot(a+(b+x))=c \cdot a+(c \cdot b+c \cdot x)=$ $(c \cdot a+c \cdot b)+d_{c \cdot a, c \cdot b} \cdot(c \cdot x)$ and $\left.c \cdot(a+(b+x))=c \cdot\left((a+b)+d_{a, b} \cdot x\right)\right)=$ $(c \cdot a+c \cdot b)+c \cdot\left(d_{a, b} \cdot x\right)$ hence $c^{\bullet} \circ d_{a, b}^{\bullet}=d_{c a, c b}^{\bullet} \circ c^{\bullet}$.

(4) d) By (4) a), $\mathfrak{J}_{E} \circ \Omega \subseteq \Omega$ hence $a^{+} \circ b^{\bullet}=a^{\circ} \circ o^{\circ} \circ b^{\bullet} \in a^{\circ} \circ\left(\mathfrak{J}_{E} \circ \Omega_{o}\right) \subseteq$ $\mathfrak{J}_{E} \circ \Omega \subseteq \Omega$.

(4) e) Let $a:=\omega(o)$ and $b:=o^{\circ} \circ a^{\circ} \circ \omega(e)$ then $b \neq o$ hence by (4) d), $a^{+} \circ b^{\bullet} \in \Omega$ and since $a^{+} \circ b^{\bullet}(o)=a=\omega(o)$ and $a^{+} \circ b^{\bullet}(e)=a+b=\omega(e)$ we have $\omega=a^{+} \circ b^{\bullet}$ because $\Omega$ is sharply 2 -transitive.

(4) f) From (4)(d), (e) it follows that $\operatorname{Aff}(E,+, \cdot)=\Omega$ hence (f) is a consequence of Theorem $1.5(6)$. (4)(g) follows from Corollary 1.7. 
Corollary 2.4. If $(E, \Omega)$ satisfies $\left(S^{\prime \prime}\right)$ then $(E, \Omega)$ is of class II or III.

Proof. The proof of (4)(a) did not use the assumption about the class of $(E, \Omega)$, hence it follows from Theorem 2.1(6)(a).

Remark 4. If in Theorem $2.3(E, \Omega)$ satisfies $\left(S^{\prime \prime}\right)$ then $(E,+, \cdot)$ is a quasidomain called the associated quasi-domain of $(E, \Omega)$.

From Theorems 2.3. and 1.5. we obtain:

Corollary 2.5. Let $(E, \Omega)$ be of class II or III then:

(1) If $(E, \Omega)$ satisfies $\left(S^{\circ}\right)$ and $\left(S^{\prime}\right)$ then $(E,+, \cdot)$ is a quasi-domain where $(E,+)$ is a K-loop and $\left(E^{*}, \cdot\right)$ is a Bol loop.

(2) If $(E, \Omega)$ satisfies $\left(S^{\prime \prime}\right)$ then $(E,+, \cdot)$ is a quasi-domain satisfying the condition (6)(a) and (7)(a) of Theorem 1.5 and therefore $\operatorname{Aff}(E,+, \cdot)=\Omega=$ $\Omega^{-1}$.

Corollary 2.6. If in a quasi-domain $(Q,+, \cdot),(Q, \cdot)$ satisfies the Bol-identity and the condition $(6)(a)$ of Theorem 1.5 then $(Q,+, \cdot)$ satisfies the condition (7) (a) of Theorem 1.5 and $(Q,+)$ is a K-loop.

Proof. By Theorem $1.6(2)(\mathrm{b}),(Q, \operatorname{Aff}(Q,+, \cdot))$ satisfies $(\mathrm{S})$ and by Corollary 1.7 the conditions (7)(a),(b) of Theorem 1.5. By Theorem 1.5(7),(9), $(Q,+, \cdot)$ also satisfies (8)(b). Hence, by Theorem 1.6(2)(a), $(Q$, Af $f(Q,+, \cdot))$ satisfies $(*)$. Thus, by Corollary $2.4,(Q, \operatorname{Aff}(Q,+, \cdot))$ is a sharply 2 -transitive permutation set of class II or III and $(Q,+)$ is a K-loop, by Theorem 2.3.

\section{Properties of the Germ of a Symmetric 2-Structure}

By Theorems 1.2. and 1.4., the theory of symmetric 2-structures is equivalent with the theory of sharply 2-transitive symmetric permutation sets and by [6] there are three classes of symmetric 2-structure $\Sigma:=\left(P, \mathfrak{G}_{1}, \mathfrak{G}_{2}, \mathfrak{K}\right)$ depending whether for $K \in \mathfrak{K}$ and $p \in K$ we have $\left|(p \perp K)_{\mathfrak{K}}\right|>1$ (Class I) or $(p \perp K)_{\mathfrak{K}}=$ $\emptyset$ (Class II) or $\left|(p \perp K)_{\mathfrak{K}}\right|=1$ (Class III). By [7], each symmetric 2-structure of class III is point-symmetric, i.e. $\forall(A, B) \in \mathfrak{K}^{2 \perp}: A \cap B \neq \emptyset$.

We have the corresponding classification of sharply 2 -transitive symmetric permutation sets $(E, \Omega)$ : Let again $o \in E$ be fixed and let $\Omega_{o}:=\{\omega \in$ $\Omega \mid \omega(o)=o\}$ be the stabilizer of the element $o$. Then $(E, \Omega)$ belongs to class I if $\left|\Omega_{o} \cap \mathfrak{J}_{E}\right|>1$, to class II if $\Omega_{o} \cap \mathfrak{J}_{E}=\emptyset$ and to class III if $\left|\Omega_{o} \cap \mathfrak{J}_{E}\right|=1$. If $(E, \Omega)$ belongs to class III then $\forall \alpha \in \Omega \cap \mathfrak{J}_{E},|F i x \alpha|=1$. This classification agrees with that given after Theorem 2.1.

If $(E, \Omega)$ is a sharply 2-transitive permutation group then the condition (S) is satisfied. By [10, Satz (11.10)] there are two types of such permutation groups characterized by

" $\forall \alpha \in \mathfrak{J}_{E}:|F i x \alpha|=1$ " hence $(E, \Omega)$ belongs to class III and by

" $\forall \alpha \in \mathfrak{J}_{E}:$ Fix $\alpha=\emptyset$ " hence $(E, \Omega)$ belongs to class II. 
By $[1, \S 5]$, to $(E, \Omega)$ there corresponds a neardomain $(E,+, \cdot)$ such that $\Omega$ is the affine group of the neardomain and $(E, \Omega)$ belongs to class III if $\operatorname{Char}(E,+, \cdot) \neq 2$ and to class II if $\operatorname{Char}(E,+, \cdot)=2$.

We can summarize:

Theorem 3.1. Let $\Sigma:=\left(P, \mathfrak{G}_{1}, \mathfrak{G}_{2}, \mathfrak{K}\right)$ be a 2-structure, $E \in \mathfrak{K}$ and $(E, \Omega):=$ $\Pi_{E}\left(P, \mathfrak{G}_{1}, \mathfrak{G}_{2}, \mathfrak{K}\right)$ the germ. Then:

(A) Let $o \in E$ be fixed, $\Omega_{o}:=\{\omega \in \Omega \mid \omega(o)=o\}, \mathfrak{J}_{E}:=\left\{\omega \in \Omega \mid \omega^{2}=i d \neq \omega\right\}$ and let $\Sigma$ be symmetric then

(1) $\Sigma$ belongs to class $I \Leftrightarrow\left|\Omega_{O} \cap \mathfrak{J}_{E}\right|>1$.

(2) $\Sigma$ belongs to class $I I \Leftrightarrow \Omega_{o} \cap \mathfrak{J}_{E}=\emptyset$.

(3) $\Sigma$ belongs to class III $\Leftrightarrow\left|\Omega_{o} \cap \mathfrak{J}_{E}\right|=1$.

(B) If $\Sigma$ is point symmetric then $(E, \Omega)$ has the following properties:

(1) $\forall a \in E \exists_{1} a^{\circ} \in \mathfrak{J}_{E}$ with Fix $a^{\circ}=\{a\}$.

(2) $\forall a, b \in E \exists_{1} c \in E$ with $c^{\circ}(a)=b$.

(3) $\forall \omega \in \Omega, \forall a \in E$ and $a^{\prime}:=\omega(a): \omega \circ a^{\circ}=a^{\prime \circ} \circ \omega$ and $\omega \circ a^{\circ} \in \Omega$.

(C) The following statements are equivalent:

(1) $\Sigma$ is double symmetric.

(2) $(E, \Omega)$ is a sharply 2-transitive permutation group.

(3) There is a neardomain $(E,+, \cdot)$ such that $(E, \Omega)$ is the affine group of the neardomain.

(D) If $\Sigma$ is double symmetric then $\Sigma$ belongs to class II or III and the following statements are equivalent:

(1) $(P, \mathfrak{B})$ with $\mathfrak{B}:=\mathfrak{G}_{1} \cup \mathfrak{G}_{2} \cup \mathfrak{K}$ is an affine plane.

(2) $(E,+, \cdot)$ is a planar nearfield

(E) If $\Sigma$ is finite then $(P, \mathfrak{B})$ is an affine plane.

Because of Theorem 3.1. we have now to focus our interest on symmetric 2-structures which are not double symmetric.

\subsection{Some Properties of a Point Symmetric 2-Structure}

Let $\Sigma:=\left(P, \mathfrak{G}_{1}, \mathfrak{G}_{2}, \mathfrak{K}\right)$ be a point symmetric 2 -structure, i.e. $\forall(A, B) \in \mathfrak{K}^{2 \perp}$ : $|A \cap B|=1$, let $E \in \mathfrak{K}$ be fixed, let $(\mathfrak{C}, \cdot):=(\mathfrak{C}, \cdot)_{E}$, let $\mathfrak{J}_{\mathfrak{K}}:=\mathfrak{J} \cap \mathfrak{K}$ and for $A \in \mathfrak{K}$ let here $A_{\mathfrak{K}}^{\perp}:=A^{\perp} \cap \mathfrak{K}$. Then we have (cf. [7]):

Theorem 3.2. (1) $\forall p \in P, \forall K \in \mathfrak{K}:\left|(p \perp K)_{\mathfrak{K}}\right|=1$.

(2) $\forall(A, B),(C, D) \in \mathfrak{K}^{2 \perp}$ with $A \cap B=C \cap D: \widetilde{A} \circ \widetilde{B}=\widetilde{C} \circ \widetilde{D}$ and if $p:=A \cap B$ then $\widetilde{p}:=\widetilde{A} \circ \widetilde{B}$ is the reflection in the point $p$.

(3) For $a \in P$ let " $\alpha_{1}: P \rightarrow P ; p \mapsto \widetilde{a p}(p)$ " and " $\alpha_{2}: P \rightarrow P ; p \mapsto \widetilde{p a}(p)$ " then $\alpha_{i}$ is the reflection in the generator $[a]_{i}$ also denoted by $\widetilde{[a]_{i}}$ and $\alpha_{i} \in \operatorname{Aut}\left(P, \mathfrak{G}_{1}, \mathfrak{G}_{2}, \mathfrak{K}\right)$.

(4) We have: $\widetilde{a}=\alpha_{1} \circ \alpha_{2}=\alpha_{2} \circ \alpha_{1}$.

(5) If $K \in \mathfrak{K}$ and $k:=K \cap[a]_{i}$ then $\alpha_{i}(K)=(k \perp K)_{\mathfrak{K}}$. 
Theorem 3.3. Let $\Sigma:=\left(P, \mathfrak{G}_{1}, \mathfrak{G}_{2}, \mathfrak{K}\right)$ be a point symmetric 2-structure. Then:

(1) $\forall A, B \in \mathfrak{K}: \widetilde{A B}\left(A_{\mathfrak{K}}^{\perp}\right)=B_{\mathfrak{K}}^{\perp}$.

(2) $\forall A, B \in \mathfrak{K} \forall C \in A_{\mathfrak{K}}^{\perp}: A \cdot C^{-1} \cdot B \in \mathfrak{K}$.

(3) $\mathfrak{K} \cdot \mathfrak{J}_{\mathfrak{K}}=\mathfrak{K}$ and $\mathfrak{J}_{\mathfrak{K}} \cdot \mathfrak{K}=\mathfrak{K}$.

Proof. (1) Let $x \in P$ and $y:=\widetilde{A B}(x)=(B x)(x A)=[B x]_{1} \cap[x A]_{2}$.

Let $a:=(x \perp A)_{\mathfrak{K}} \cap A$ (hence $\left.(x \perp A)_{\mathfrak{K}}=(a \perp A)_{\mathfrak{K}}\right)$, let $\alpha_{2}:=\widetilde{[a]_{2}}$ be the reflection in the generator $[a]_{2}$ (hence by Theorem $3.2(5), \alpha_{2}\left((a \perp A)_{\mathfrak{K}}\right)=$ $A)$ and let $b:=[a]_{2} \cap B$. Then $\widetilde{A B}(a)=b$ and again by Theorem $3.2(5)$, $\alpha_{2}(B)=(b \perp B)_{\mathfrak{K}}$. Since $x=[x]_{1} \cap(a \perp A)_{\mathfrak{K}}, \alpha_{2}(x)=[x]_{1} \cap A=x A$ hence $\alpha_{2}\left([x]_{2}\right)=[x A]_{2}$ and $\alpha_{2}(B x)=[B x]_{1} \cap[x A]_{2}=y$ implying $\alpha_{2}(B)=\alpha_{2}(\overline{b, B x})=\overline{b, y} \perp B$. Thus $y \in(b \perp B)_{\mathfrak{K}}$, i.e. $\widehat{A B}\left(A_{\mathfrak{K}}^{\perp}\right)=B \frac{\perp}{\mathfrak{K}}$.

(2) Let $C \in A_{\mathfrak{K}}^{\perp}$ hence $A \cdot C^{-1} \cdot A=C$ then by $(1), D:=\widetilde{A B}(C)=A \cdot C^{-1} \cdot B \in$ $B_{\mathfrak{K}}^{\perp} \subseteq \mathfrak{K}$.

(3) Let $A \in \mathfrak{K}$ and $B \in \mathfrak{J}_{\mathfrak{K}}$ hence $B=B^{-1} \in E^{\perp}$. Then by $(2), A \cdot B^{-1} \cdot E=$ $A \cdot B \in \mathfrak{K}$. By Theorem 1.4.(3), $\mathfrak{K}=\mathfrak{K}^{-1}$ and so $\mathfrak{K} \cdot \mathfrak{J}_{\mathfrak{K}}=\mathfrak{K}$ implies $\mathfrak{J}_{\mathfrak{K}} \cdot \mathfrak{K}=\mathfrak{K}$.

\subsection{A Representation of a Point Symmetric 2-Structure by a Quasi-Domain}

In the point symmetric 2-structure $\Sigma:=\left(P, \mathfrak{G}_{1}, \mathfrak{G}_{2}, \mathfrak{K}\right)$ let $E \in \mathfrak{K}$ and $(o, e) \in$ $\left(\begin{array}{l}E \\ 2\end{array}\right)$ be fixed. Let $(E, \Omega):=\Pi_{E}(\Sigma)$ be the germ of $\Sigma$ (by Theorem 1.4, $(E, \Omega$ ) is a sharply 2-transitive symmetric permutation set) and let $(E,+, \cdot)$ be the double-loop-derivation of $(E, \Omega)$ in the points $(o, e)$ (cf. Sect. 2.2). By applying the germ operation $\Pi_{E}$ onto $\Sigma$ we obtain from Theorems 3.3.(3) and Corollary 2.5.(2):

Theorem 3.4. The germ $(E, \Omega):=\Pi_{E}(\Sigma)$, has the following properties:

(1) $(E, \Omega)$ satisfies besides $(S)$ also $\left(S^{\prime \prime}\right)$ hence $(E,+, \cdot)$ is a quasi-domain.

(2) $(E, \Omega)$ is of class III.

(3) Any two points $a, b \in E$ have exactly one midpoint $m \in E$, i.e. there is exactly one involution $\mu \in \mathfrak{J}_{E}$ with Fix $\mu=m$ and $\mu(a)=b$.

Now we state our main result on point symmetric 2-structures:

Theorem 3.5. If $\Sigma$ is a point symmetric 2-structure and $(E, \Omega)=\Pi_{E}(\Sigma)$ the germ then $(E,+, \cdot)$ is a quasi-domain of char $E \neq 2$ and:

(1) $\forall a . b, c, x \in E: c\left(d_{a, b} x\right)=\left(c d_{a, b}\right) x$.

(2) $(E,+)$ is a uniquely 2-divisible K-loop where o is the neutral element.

(3) $\left(E^{*}, \cdot\right)$ is a Bol loop where $e$ is the neutral element.

(4) $\Omega=\operatorname{Aff}(E,+, \cdot)$.

Conversely: Let $(E,+, \cdot)$ be a quasi-domain of char $E \neq 2$ satisfying the conditions (1) and (3) and let $\Omega=\operatorname{Aff}(E,+, \cdot)$ then the chain derivation $\kappa((E, \Omega))$ is a point symmetric 2-structure. 
Proof. " $\Rightarrow$ " By Theorem 1.4.(4), $(E, \Omega)$ is a sharply 2-transitive symmetric permutation set and so, by Theorem 2.2., $(E,+)$ is a uniquely 2-divisible $\mathrm{K}$ loop. By Theorem 3.3.(3), $\mathfrak{K} \cdot \mathfrak{J}_{\mathfrak{K}}=\mathfrak{K}$ hence $\Omega \circ \mathfrak{J}_{E}=\Omega$, i.e. $(E, \Omega)$ satisfies $\left(S^{\prime \prime}\right)$, hence the other statements follow from Theorem $2.3(4)$.

" $\Leftarrow$ " The condition $(1)$ implies by Theorem $1.5(6)$ that $(E, \Omega)$ is a sharply 2 -transitive permutation set. The condition (3) implies by Theorem 1.6(2)(b) that $(E, \Omega)$ is symmetric. Therefore by Theorem $1.4(4) \kappa((E, \Omega))$ is a symmetric 2-structure. Moreover by Corollary $1.7,(1)$ and $(3)$ imply that $(E,+, \cdot)$ satisfies the condition (7)(a) of Theorem 1.5, hence also the condition (8)(b) of Theorem 1.5. Now if $a^{+} \circ b^{\bullet} \in \Omega_{o} \cap \mathfrak{J}_{E}$ then $o=a^{+} \circ b^{\bullet}(o)=a+o=a$ hence $a^{+} \circ b^{\bullet}=b^{\cdot} \in \mathfrak{J}_{E}$ implies $b(b x)=x$ in particular $b \cdot b=e$ and $b \neq e$. Therefore $\Omega_{o} \cap \mathfrak{J}_{E}=\left\{(-e)^{\bullet}\right\}$, by $[2,(1.4)(\mathrm{a})]$ and by Theorem $3.1(\mathrm{~A})(3), \kappa((E, \Omega))$ is of class III.

Open Access. This article is distributed under the terms of the Creative Commons Attribution License which permits any use, distribution, and reproduction in any medium, provided the original author(s) and the source are credited.

\section{References}

[1] Karzel, H.: Zusammenhänge zwischen Fastbereichen, scharf zweifach transitiven Permutationsgruppen und 2-Strukturen mit Rechtecksaxiom. Abh. Math. Semin. Univ. Hamburg 32, 191-206 (1968)

[2] Kist, G.: Theorie der verallgemeinerten kinematischen Räume. Habilitationsschrift, Technische Universität München (1980) Printed in: TUM-MATH-10-86M11-250/1- FMI

[3] Kiechle, H.: Theory of K-Loops. In: Lecture Notes in Mathematics 1778, Springer, Berlin (2002)

[4] Karzel, H., Kosiorek, J., Matraś, A.: Properties of auto-and antiautomorphisms of maximal chain structures and their relations to i-perspectivities. Result Math. 50, 81-92 (2007)

[5] Karzel, H., Kosiorek, J., Matraś, A.: Automorphisms of symmetric and double symmetric chain structures. Result Math. 55, 401-416 (2009)

[6] Karzel, H., Kosiorek, J., Matraś, A.: Symmetric 2-structures, a classification. Result Math., submited

[7] Karzel, H., Kosiorek, J., Matraś, A.: Point symmetric 2-structures. Result Math. 59, 229-237 (2011)

[8] Kühlbrandt, H.: Algebraisierung scharf 2-fach transitiver Permutationsmengen durch Quasibereiche. J. Geom. 17, 7-23 (1981)

[9] Karzel, H.: Loops related to geometric structures. Quasigroups Relat. Syst. 15, 47-76 (2007)

[10] Karzel, H.: INZIDENZGRUPPEN. In: Vorlesungsausarbeitung von I. Pieper und K. Sörensen, Hamburg (1965) 
Helmut Karzel

Zentrum Mathematik

T.U. München

80290 Munich, Germany

e-mail: karzel@ma.tum.de

Jarosław Kosiorek and Andrzej Matraś

Faculty of Mathematics and Computer Science

University of Warmia and Mazury

Słoneczna 54, 10-710 Olsztyn, Poland

e-mail: kosiorek@matman.uwm.edu.pl;

matras@uwm.edu.pl

Received: December 6, 2011.

Accepted: October 12, 2012. 